\title{
Developmental relations between motivation types and physical activity in elementary school children (1)
}

COACHING / EDUCATION / EXERCISE IS MEDICINE / MENTAL HEALTH / PA PROMOTION / PHYSICAL

EDUCATION / SPORTS AND SOCIETY

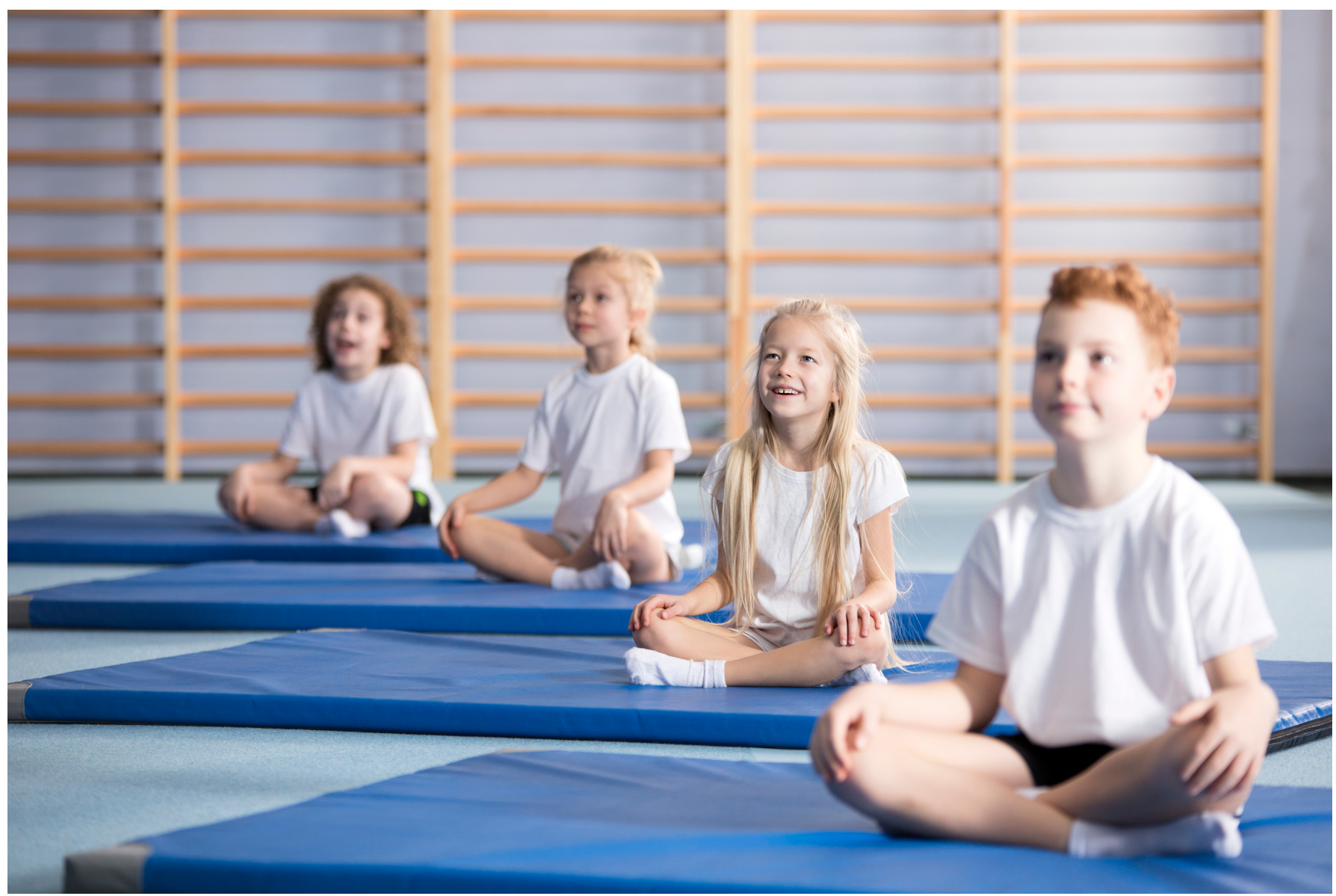

\section{Moreillon M}

Swiss Olympic Medical Center, Hôpital de La Tour, Meyrin, Genève

\section{Abstract (of original paper by Chanal et al.)}

Objectives: The objectives of this study were to observe the developmental trajectories of motivation types among young children from 8 to 12 years using a more comprehensive scale of physical education motivation. We also tested the relations between these trajectories and objective physical activity during 
this period.

Design: Students in grades 5-7 ( $\mathrm{n}=1202 ; 51.2 \%$ boys) were recruited from 17 elementary schools. Three cohorts completed the motivation questionnaire four times and objective physical activity was measured up to four times over a two years school period.

Method: Measurement invariance of the scale was tested across cohorts and occasions. Multiple group multiple cohort growth models were estimated to determine motivation types trajectories. Regression models were then built to predict children's slope of MVPA during this period.

Results: We provided strong measurement invariance to a new and more comprehensive scale of PE motivation. Latent growth curve modeling indicated trajectories that decrease on average for all forms of motivations at this early age. Results also revealed some relations between motivation's scores and objective physical activity trajectories, especially with autonomous motivation.

Conclusions: Our study revealed the earliest decline of motivation towards physical education to have ever been highlighted in elementary school children. Relations between trajectory of intrinsic stimulation and PA behavior permitted us to highlight the possible role of autonomous motivation in minimizing the decline of children's PA behavior during PE lesson.

Physical activity (PA) has a significant impact in health promotion and prevention of numerous noncommunicable diseases already in the youth [2-4]. To promote PA in this population, public heath recommendations advise that at least $50 \%$ of physical education (PE) lessons are spent in moderate to vigorous physical activity (MVPA) [5]. However, despite PE programs, children and adolescents do not reach these MVPA goals [6-8]. Moreover they demonstrate a decline in PA levels as early as in the elementary schools [9]. This is of concern because PA levels during childhood and adolescence are significantly related to an active lifestyle during young adulthood [10], which in turn promotes health at later ages. Given the fact that changes in motivation seem to predict trajectories in leisure-time PA across ages [11], the present study aimed at investigating (a) the developmental trajectories of motivation types for PE sessions in 8 to 12 years old students, and (b) the potential relations between motivation and objective PA trajectories during elementary school PE lessons.

Seventeen different elementary schools from the Canton of Geneva, Switzerland, were included. Motivation and objective PA data from 1202 volunteer students (51.2\% boys, 48.8\% girls evenly distributed from grades 5 to 7) were longitudinally monitored four times during the 2012 and 2013 academic years. Factor loadings of the time scores were normalised in order to account for an accelerated longitudinal design with three evenly distributed cohorts of students from grades 5 to 7. PE lessons were taught in mixed-gender classes with no specific instructions to PE teachers. Accelerometer recorded lessons were selected randomly and questionnaires were completed by students in non-PE classes. Motivation types described in the Self-determined Theory [12] were assessed using a 33-item questionnaire extracted from different scales specifically used in physical education settings [13-15]. Broadly, these items were divided in eight subscales including different subtypes of (i) intrinsic motivation, which is the most autonomous 
form, referring to engaging in an activity for its inherent pleasure and satisfaction to stimulation (sensory pleasure), learning, or accomplishment (pleasure of surpassing); (ii) extrinsic motivation, which is an extrinsically motivated behaviour to attain desirable goals or to avoid negative consequences, thus including an approach (e.g. seeking reward) and avoidance (e.g. avoiding punishment or shame) dimension; as well as (iii) amotivation, which is the lack of intention and willingness to perform a behaviour.

Overall, the authors observed decreasing motivation trajectories in all intrinsic motivation subtypes (motivation for the pleasure of learning, surpassing, and sensory pleasure) and in three extrinsic motivation subtypes (grossly motivation for an identified value in undertaking PA, and motivation for external contingencies such as rewards, or avoiding punishments, guilt or shame). This was valid for any of the 8 to 12 years old study groups. On the other hand, a positive relation was found between intrinsic motivation and MVPA, highlighting the role of pleasure in PA behaviour during PE. Likewise, changes in the intrinsic motivation subtype for sensory pleasure were positively related to changes in MVPA. Thus, positive changes in intrinsic motivation could positively impact PA behaviour in elementary school children. Few relationships were found between extrinsic motivation and MVPA. Only one extrinsic motivation subtype seemed to be positively related to MVPA in the approach dimension (seeking reward), and negatively in the avoidance dimension (avoiding punishment). Thus, motivation for seeking reward could act as a positive determinant of PA behaviour in PE lessons, whereas motivation for avoiding punishment could have a deleterious effect.

Despite a large sample of elementary school children, detailed motivation type analysis including the approach (seeking reward) and avoidance (avoiding punishment, guilt or shame) distinction in extrinsic motivation subtypes, and objective measurement of PA, some limitations in the study exist as underlined by the authors. First, the data is limited to a specific socio-demographic and cultural setting. These factors could have important implications on the outcomes. Therefore, they should be verified by similar studies in different populations and cultures before concluding to prevention or intervention strategies. Second, since complex statistics were implemented in the analyses, the risk of accidental bias cannot be entirely excluded. Third, PA behaviour was reflected by the percentage of time spend in MVPA during the entire PE lessons. Nevertheless, the structure of the lessons implies periods in which students cannot behave autonomously (warm-up, instructions, etc.). Consequently, PA intensities in the course of PE lessons cannot only be attributed to the students' motivation. Finally, since the study used a new scale to evaluate students' motivation for PE it might be difficult to strictly compare the results with other studies without prior cross validation.

In conclusion, the present study adds to previous work $[11,16,17]$ by showing that the decline in students' motivation for PE already takes place in 8 to 12 years old, specifically for subtypes of both intrinsic and extrinsic motivation. In addition, the authors found relations between these declines in motivation trajectories and objective PA behaviours.

As prior work observed that positive experiences and motivation for PE were shown to be associated with positive effects on PA-related behaviours [11,18-20], the authors of the present study [1] identify relations between different motivation subtypes of the Self-determined Theory [12] and MVPA. The intrinsic motivation subtype for sensory pleasure and the extrinsic motivation subtype for reward (approach) were positively related to MVPA, while the extrinsic motivation subtype for avoiding punishment (avoidance) was negatively related to it. This is crucial in order to understand and target the different factors 
contributing to the decline of motivation for PE across ages, as well as to design more efficient programs to prevent it.

The present study performed in young age groups emphasizes that such programs must be implemented early in elementary school children, and that they should encourage intrinsic motivation for pleasure such as sensory pleasure and eventually extrinsic motivation for reward. While it is essential to sensitize PE teachers to the importance of motivation for PE in young children, additional work is needed to better understand the key modifiable factors underlining the decline in PA-related behaviours and adaptive motivation across age, and implement the best possible promotion strategies for an active lifestyle.

\section{Keypoints}

- Physical activity levels during childhood and adolescence are significantly related to an active lifestyle during young adulthood.

- Declines in students' motivation for physical education exist as early 8 to 12 years old and are related with declines in physical activity.

- Among others, prevention of decline in motivation for physical education must target changes in specific motivation types in younger children already.

- Extrinsic motivation subtype for avoiding punishment seems to be negatively related to changes in physical activity.

- Intrinsic motivation for sensory pleasure and extrinsic motivation for reward seem to be positively related to changes in physical activity.

\section{Corresponding author}

Maxime Moreillon, MD

La Tour Sport Medicine

Swiss Olympic Medical Center

Avenue J.-D. Maillard 3

1217 Meyrin (Genève)

Tel: 0227197775

E-mail: maxime.moreillon@latour.ch $\mathrm{x}$

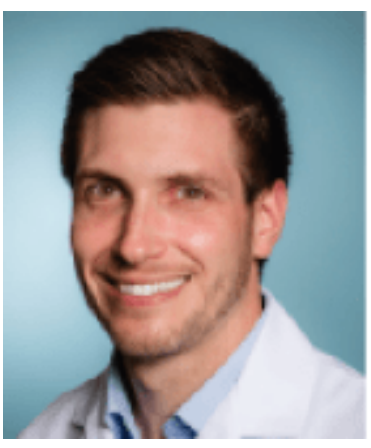

\section{References}

1. Chanal J, Cheval B, Courvoisier DS, Paumier D. Developmental relations between motivation types and physical activity in elementary school children. Psychology of Sport and Exercise. 2019;43:233-42.

2. Andersen LB, Harro M, Sardinha LB, Froberg K, Ekelund U, Brage S, et al. Physical activity and clustered cardiovascular risk in children: a cross-sectional study (The European Youth Heart Study). Lancet (London, England). 2006;368(9532):299-304.

3. Kimm SY, Glynn NW, Obarzanek E, Kriska AM, Daniels SR, Barton BA, et al. Relation between the changes in physical activity and body-mass index during adolescence: a multicentre longitudinal study. Lancet (London, England). 2005;366(9482):301-7. 
4. Kasa-Vubu JZ, Lee CC, Rosenthal A, Singer K, Halter JB. Cardiovascular fitness and exercise as determinants of insulin resistance in postpubertal adolescent females. The Journal of clinical endocrinology and metabolism. 2005;90(2):849-54.

5. Centers for Disease Control and Prevention. School health guidelines to promote healthy eating and physical activity. 60(5): MMWR; 2011. p. 30-1.

6. Colley RC, Garriguet D, Janssen I, Craig CL, Clarke J, Tremblay MS. Physical activity of Canadian children and youth: accelerometer results from the 2007 to 2009 Canadian Health Measures Survey. Health reports. 2011;22(1):15-23.

7. Glickman D, Parker L, Sim LJ, Del Valle Cook H, Miller EA. Committee on Accelerating Progress in Obesity Prevention; Food and Nutrition Board; Institute of Medicine. Accelerating Progress in Obesity Prevention: Solving the Weight of the Nation. Washington (DC): National Academies Press (US); 2012.

8. Sallis JF, McKenzie TL, Beets MW, Beighle A, Erwin H, Lee S. Physical education's role in public health: steps forward and backward over 20 years and HOPE for the future. Research quarterly for exercise and sport. 2012;83(2):125-35.

9. Cheval B, Courvoisier DS, Chanal J. Developmental trajectories of physical activity during elementary school physical education. Preventive medicine. 2016;87:170-4.

10. Kjonniksen L, Anderssen N, Wold B. Organized youth sport as a predictor of physical activity in adulthood. Scandinavian journal of medicine \& science in sports. 2009;19(5):646-54.

11. McDavid L, Cox AE, McDonough MH. Need fulfillment and motivation in physical education predict trajectories of change in leisure-time physical activity in early adolescence. Psychology of Sport and Exercise. 2014;15(5):471-80.

12. Ryan RM, Deci EL. Self-determination theory and the facilitation of intrinsic motivation, social development, and well-being. The American psychologist. 2000;55(1):68-78.

13. David M, Vannessa T. A Modification to the Behavioural Regulation in Exercise Questionnaire to Include an Assessment of Amotivation. 2004;26(2):191.

14. Li F. The exercise motivation scale: Its multifaceted structure and construct validity. Journal of Applied Sport Psychology. 1999;11(1):97-115.

15. Pelletier LG, Rocchi MA, Vallerand RJ, Deci EL, Ryan RM. Validation of the revised sport motivation scale (SMS-II). Psychology of Sport and Exercise. 2013;14(3):329-41.

16. Jaakkola T, Wang J, Yli-Piipari S, Liukkonen J. A multilevel latent growth modelling of the longitudinal changes in motivation regulations in physical education. Journal of sports science \& medicine. 2015;14(1):163-71.

17. Ntoumanis N, Barkoukis V, Thøgersen-Ntoumani C. Developmental trajectories of motivation in physical education: Course, demographic differences, and antecedents. Journal of Educational Psychology. 2009;101(3):717-28.

18. Hagger MS, Chatzisarantis NLD, Barkoukis V, Wang CKJ, Baranowski J. Perceived Autonomy Support in Physical Education and Leisure-Time Physical Activity: A Cross-Cultural Evaluation of the TransContextual Model. Journal of Educational Psychology. 2005;97(3):376-90.

19. Yli-Piipari S, Leskinen E, Jaakkola T, Liukkonen J. Predictive role of physical education motivation: the developmental trajectories of physical activity during grades 7-9. Research quarterly for exercise and sport. 2012;83(4):560-9.

20. Taylor IM, Ntoumanis N, Standage M, Spray CM. Motivational predictors of physical education students> effort, exercise intentions, and leisure-time physical activity: a multilevel linear growth analysis. Journal of sport \& exercise psychology. 2010;32(1):99-120. 